\title{
Detecting Phone Theft Using Machine Learning
}

\author{
Xinyu Liu \\ UC Berkeley \\ xinyuliu@berkeley.edu
}

\author{
David Wagner \\ UC Berkeley \\ daw@cs.berkeley.edu
}

\author{
Serge Egelman \\ UC Berkeley/ICSI \\ egelman@cs.berkeley.edu
}

\begin{abstract}
Millions of smartphones are stolen in the United States every year, putting victims' personal information at risk since many users often do not lock their phones. To protect individuals' smartphones and the private data stored on them, we developed a system that automatically detects pickpocket and grab-and-run theft, in which a thief grabs the phone from a victim's hand then runs away. Our system applies machine learning to smartphone accelerometer data in order to detect possible theft incidents. Based on a field study and simulated theft scenarios, we are able to detect all thefts at a cost of 1 false alarm per week. Given that many smartphone users refuse to enable screen locking mechanisms over complaints that it takes too long to unlock their devices, our system could be used in conjunction with these systems in order to drastically decrease the number of times a user is asked to provide a lock code. That is, our system could be used to prompt smartphone users for PINs or passcodes only when theft events have been detected.
\end{abstract}

\section{CCS Concepts}

- Security and privacy $\rightarrow$ Mobile and wireless security; Biometrics;

\section{Keywords}

Usable security; machine learning; smartphone theft detection

\section{INTRODUCTION}

According to Consumer Reports, 2.1 million smartphones were stolen in the United States in 2014 [4], and the Pew Research Center's Internet \& American Life Project reported in 2012 that nearly one third of mobile phone users have experienced a lost or stolen device [2]. People can lock their phones to mitigate the risks of phone theft, but some find this inconvenient, as it requires unlocking the phone every time it is needed. As a result, about $40 \%$ of smartphone users do not lock their phones, which allows thieves to gain access to the victims' personal informationinformation that most users underestimate the sensitivity of [7]. Our goal is to increase the usability of phone locking, and therefore its adoption, by only forcing users to provide unlock codes when there is a reasonable chance that the device is being used by someone other than the rightful owner. To that end, we developed a method to automatically detect pickpocket and graband-run smartphone theft by training a binary classifier to

Permission to make digital or hard copies of all or part of this work for personal or classroom use is granted without fee provided that copies are not made or distributed for profit or commercial advantage and that copies bear this notice and the full citation on the first page. Copyrights for components of this work owned by others than the author(s) must be honored. Abstracting with credit is permitted. To copy otherwise, or republish, to post on servers or to redistribute to lists, requires prior specific permission and/or a fee. Request permissions from Permissions@acm.org.

ICISS '18, April 27-29, 2018, Jeju, Republic of Korea

(C) 2018 Copyright is held by the owner/author(s). Publication rights licensed to ACM.

ACM ISBN 978-1-4503-6421-8/18/04\$15.00

https://doi.org/10.1145/3209914.3209923 recognize the movements that are specific to theft and use it to monitor accelerometer data in the background. When theft is detected, our system can signal the device to lock the screen or take other actions. Thus, our theft detector offers another layer of protection against smartphone theft, as well as decreases the number of times that legitimate users will be asked to explicitly unlock their device screens under ordinary circumstances. Because our theft detection software results in fewer explicit unlock prompts, it increases the usability of current smartphone locking mechanisms, and is therefore likely to increase overall adoption. Compared to post hoc security software, like kill switches and remote erase, which retroactively protects users' privacy, our system operates in real-time and may therefore better protect users' private information.

There are multiple ways that a phone might be stolen. We focus specifically on grab-and-run theft, where the thief snatches the phone out of the user's hand and runs away, and pickpocket theft, where the thief steals the phone from the user's pocket or bag and runs away. This creates an abrupt and unusual movement pattern, which we show can be detected from analysis of accelerometer sensor data. We do not attempt to detect other forms of theft, such as where the phone is left unattended and the thief walks off with it, or where the phone is lost or left behind somewhere. Consequently, our scheme cannot offer comprehensive protection against all forms of theft, but we hope that it will be useful nonetheless.

We measure the efficacy of our scheme by gathering two datasets. First, we simulated three types of phone theft: grab-and-run while the victim is standing still, grab-and-run while the victim is walking at a constant speed, and pick-pocket theft. We collect accelerometer sensor readings during the simulated thefts; these serve as known positives. Secondly, we conducted a field study where we collected 3 weeks of sensor readings from the phones of 53 participants during their everyday activities. No phone was stolen during the field study, so this dataset serves as known negatives. We use this to train a classifier and then evaluate its detection rate and false positive rate. Our best classifier produces 1 false alarm per week on average, while detecting $100 \%$ of simulated thefts.

Our contributions are:

(1) We conducted a user study and collected a large dataset of smartphone sensor data while devices were being used in the real world.

(2) We devise features and methods to detect two common types of theft, and we show that our system can detect these types of thefts with few false positives.

\section{RELATED WORK}

Many researchers have studied using smartphone sensors for continuous user authentication. The benefit of continuous user authentication is that it happens unobtrusively, without requiring any action from users. Continuous authentication could serve as a mitigation for theft: if the phone can detect rapidly enough that it 
is no longer being used by the rightful owner, it could lock itself to prevent the thief from accessing sensitive data on the phone.

One approach is to use smartphone accelerometer data for gait recognition. These systems often extract some features from the sensor data and then apply machine learning. Derawi et al. achieved an equal error rate of 20\% [5]. "Equal error rate" is a measure of accuracy where the system is tuned so the false accept rate and false reject rates are equal, and then that error rate is reported. Primo et al. show that accelerometer-based gait authentication is somewhat dependent on the position in which the phone is held, which is a challenge for deploying gait authentication outside of a laboratory environment [13]. They showed how to infer the position of the phone (in the person's hand vs. in a pocket) with $85 \%$ accuracy, and they showed how to use this information to increase the accuracy of user authentication to $70-80 \%$. They do not report performance as equal error rate. Juefei-Xu et al. show that the pace at which people walk also affects the sensor readings, and it is possible to improve accuracy by first identifying the pace at which the user is walking, then using a model tailored towards that pace [10]. Their system achieved an equal error rate of $4-8 \%$ (depending on the pace); or a false reject rate of $0.5-5 \%$ at a false accept rate of $0.1 \%$. Kwapisz et al. generalized gait authentication to cover not just walking but also jogging and ascending and descending stairs [11] and achieved false reject rates of $10-15 \%$ at a false accept rate of about $5 \%$.

It is not clear whether gait recognition is sufficient on its own for deployable user authentication. One limitation is that it can only attempt to authenticate the user while the user is walking; when the user is still, it cannot infer user identity. Another limitation is that the error rate is still fairly high: if the classifier is run continuously, once per second, even a false reject rate as low as $0.5 \%$ will cause hundreds or thousands of false rejections per week. Thus, gait recognition might need to be combined with other methods to yield a deployable defense against theft.

Our work builds on the methods previous researchers have used to process sensor data and extract features. The accelerometer sensor provides raw data in the form of $X, Y, Z$ accelerations; it is useful to also compute the magnitude $M=\sqrt{X^{2}+Y^{2}+Z^{2}}$ of the acceleration, as that is independent of the direction of the acceleration. Prior papers have used several methods for cleaning the raw accelerometer data, including interpolation and resampling to deal with irregularly sampled data and a weighted moving average filter to mitigate sensor noise. These schemes typically divide the resulting time series into windows, each window containing about a second of sensor data. For instance, Primo et al. used overlapping windows, with each window containing 100 samples and having an overlap of 50 samples with the next window; Derawi et al. and Juefei-Xu et al. used nonoverlapping windows, which were about 1 second in width. Derawi et al. and Juefei-Xu et al. used the sensor readings as the features, while Primo et al. and Kwapisz et al. computed handcrafted features from the readings, where each feature records a summary statistic on the sensor readings in the window (e.g., mean, minimum, maximum, standard deviation, number of zero crossings, etc.).

Feng et al. investigated using the unique way the user picks up their phone as a biometric for user authentication [8]. They achieved an equal error rate of $6-7 \%$. They used the smartphone accelerometer, gyroscope, and magnetometer; in our work, we avoid the gyroscope sensor, as its power consumption is significantly higher than the accelerometer, and therefore is currently not a practical solution for continuous authentication.

Mare et al. developed a continuous authentication system where the user wears a smartwatch or bracelet, which is used to authenticate the user to their computer [12]. Their continuous authentication scheme works well and could plausibly be used to authenticate to a smartphone, but it requires users to wear a bracelet; in contrast, we seek solutions that do not require the user to carry or wear any additional devices.

The most closely related work is by Chang et al., who used the way that each person took their phone out of their bag or pocket as a form of biometric authentication [3]. They use accelerometer and gyroscope data to detect when the user picks up their phone, and then they apply dynamic time warping and boosting to determine whether the pickup motion matches known templates from the owner of the phone. Their system achieves a $10 \%$ false positive rate and a $5.5 \%$ false negative rate, which are relatively high, considering that users may pick up their phones dozens of times each day.

The prior work focuses on authenticating the user. In contrast, we take a different approach: we attempt to detect the specific motion pattern that occurs during a grab-and-run or pickpocket theft. The benefit of biometric authentication is that it provides a comprehensive way to detect theft, regardless of the way the phone was stolen; however, as summarized above, the false positive rates of existing schemes are fairly high. Our scheme is limited to detecting two particular types of theft, but achieves far lower false positive rates. Our classifier is also user-independent and does not require obtaining training data from each user; we use the same classifier for all users.

\section{METHODOLOGY}

In this section, we describe the data collection software, which we used to gather positive and negative data for our training and test sets as well as the feature extraction scheme, and other classifier design decisions.

\subsection{Data Collection}

\subsubsection{Software}

We used an Android application to record data from the smartphone's 3-axis accelerometer, which then encrypted it and stored it in the cloud. We acquired sensor data at the highest sampling rate supported by the phone using SENSOR_DELAY_FASTEST [1]. On most devices, including the one we used for the simulated theft experiment, the sampling rate is $100 \mathrm{~Hz}$.

\subsubsection{Simulated Theft Experiment}

Because data from real thefts is hard to obtain, we simulated three types of common smartphone theft scenarios, based on campus police alerts that we receive, with one researcher acting as a smartphone user and another one playing the role of a thief. The three theft scenarios are as follows:

(1) The user stands still and holds the phone with one hand as she is using the device, for instance reading a text; the thief approaches from behind, grabs the phone with both hands and runs away in the forward direction.

(2) The user holds the phone in front of her with one hand while walking at a constant speed; the thief approaches 
from behind, grabs the phone with both hands and runs away in the forward direction.

(3) The third scenario simulates pick-pocket thefts. The user places the phone in the back pocket of their pants and stands still; the thief approaches from behind, steals the phone from user's pocket and runs away in the forward direction.

We collect 40 instances of each scenario, for a total of 120 trials. Data collection was split across four sessions, each consisting of 10 trials per scenario, with different researchers acting as the victim and thief in these sessions. We ran the experiment on flat ground in an open space, so the experiment was not interrupted. We also made sure that the thief ran at least 40 feet after gaining possession of the victim's phone. We used a Nexus 5X smartphone (InvenSense MPU6515 embedded accelerometer) with Android version 7.1.1 for data collection in our simulated theft experiment. Figure 1 plots one example of accelerometer readings from a single simulated theft. We collected an additional set of 120 trials, 40 instances from each scenario using a Nexus 6P smartphone with Android version 6.0.1 to demonstrate our classifier can generalize to different smartphone models.

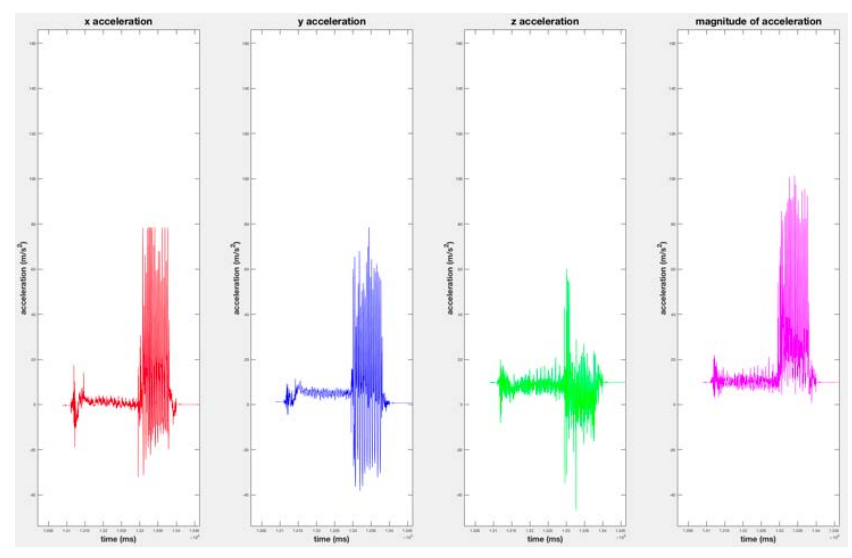

Figure 1. The $X, Y, Z$ and magnitude of acceleration, respectively, from one simulated theft instance.

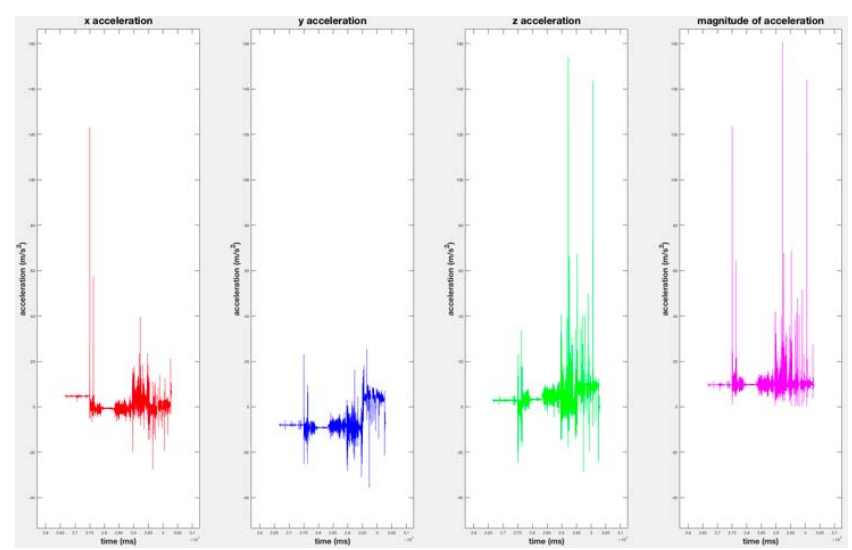

Figure 2. The $\mathrm{X}, \mathrm{Y}, \mathrm{Z}$ and magnitude of acceleration, respectively, during normal usage at an arbitrary time period.

\subsubsection{Field Study}

We performed a field study to gather data from ordinary smartphone users while they perform everyday activities, including walking, running, driving, possibly exercising, and anything else that occurred during their lives over the field study period. We obtained approval from the University of California, Berkeley IRB (Institutional Review Board) for this study. The study was conducted in the Bay Area of the United States from September to December 2016. None of the participants experienced a phone theft during the study interval, so we were able to use the accelerometer data collected from the user study as negative samples for our machine learning algorithms (i.e., instances of non-theft activity).

We posted a recruitment advertisement on Craigslist in September 2016. We only recruited participants who used an Android smartphone with version 5.0 and above. After obtaining their consent, we installed our data collection application on their phones and collected data for a three-week period. The application ran in the background and collected accelerometer sensor readings continuously, 24 hours a day. We contacted participants weekly to make sure their phones were functioning correctly and troubleshoot any data collection issues. Each participant was paid $\$ 150$ for their participation.

The study was divided across 3 rounds; each round lasted 3 consecutive weeks. A total of 55 participants were recruited, and 53 out of the 55 subjects completed the study. In the first round, 2 of the 18 participants did not complete the study. Detailed demographic information about the participants of this user study is listed in Table 1. In aggregate, they used 21 different smartphone models from 6 different manufacturers and 8 different Android versions. This ensures the dataset containing diverse devices, and the classifiers are not specific to a particular type of smartphone or Android version. We also asked participants how they typically carried their phone, when it was not in their hands; 33 reported keeping it in their pockets, 9 in their purses, 12 in multiple locations (e.g., pocket or purse, pocket or backpack), and 1 did not respond. During the study, the subjects carried their phones while performing daily activities, for example walking, driving, running and possibly exercising. Data from a wide variety of real-world activities ensures the generalizability of the classifiers trained on it.

Table 1. Participants' demographic information.

\begin{tabular}{|c|c|c|c|c|c|}
\hline & Male & Female & $\begin{array}{c}\text { Age 20- } \\
\mathbf{2 9}\end{array}$ & $\mathbf{3 0 - 3 9}$ & $\mathbf{4 0 +}$ \\
\hline R1 & 5 & 11 & 8 & 6 & 2 \\
\hline R2 & 10 & 8 & 7 & 7 & 4 \\
\hline R3 & 11 & 8 & 9 & 4 & 6 \\
\hline Total & 26 & 27 & 24 & 17 & 12 \\
\hline
\end{tabular}

\subsection{Feature Extraction}

Our theft scenarios all involved a sudden movement of the phone, which causes a large acceleration. Therefore, as a first filtering step, we filtered the data to focus on times near when a large motion occurs. In particular, our classifier is activated when the magnitude of acceleration $M$ exceeds $40 \mathrm{~m} / \mathrm{s}^{2}$. We extract a onesecond window before the activation time and a $n$-second window after the activation time, compute features on each of these windows, and use them for classification. We vary $n$ from 1 to 7 to obtain the best classification accuracy. We chose a threshold of $40 \mathrm{~m} / \mathrm{s}^{2}$ as all of our simulated thefts experienced accelerations exceeding that threshold when the phone was initially grabbed by the thief. 
We first identified 16 candidate features, 8 features for each of the two windows. In particular, we computed the minimum, maximum, mean, standard deviation, root mean square, arc length, product of arc length and standard deviation, and mean absolute value, each computed on the magnitude values $(M)$ within the window. We chose to only compute these features on the magnitude of the acceleration, and not the $X, Y$ and $Z$ components, because the magnitude is non-directional and thus more robust to changes in the orientation of the phone. We then visualized the distribution of these features for the two classes and applied feature selection techniques to choose a subset of features that yield good performance. We removed the minimum and mean absolute value from the feature list because removing them did not affect the performance of the classifiers. As a result, we extract a 12-dimensional feature vector, 6 features from the before-window and 6 from the after-window, every time the detector is triggered.

Let $M_{1}, \ldots, M_{k}$ denote the time series of acceleration magnitudes within the window. The features are computed as follows:

- $\quad$ Maximum: the maximum value of the magnitude within the window, i.e., $\max \left(M_{1}, \ldots, M_{k}\right)$.

- Mean: the average value of magnitude in a window, i.e., $\bar{M}=\left(M_{1}+\cdots+M_{k}\right) / k$.

- $\quad$ Standard deviation: the standard deviation of magnitude values in a window, i.e., $\left(\sum_{i=1}^{k}\left(M_{i}-\bar{M}\right)^{2}\right)^{1 / 2} /(k-$ $1)^{1 / 2}$.

- $\quad$ Root mean square: the RMS of magnitude values in a window, i.e., $\left(M_{1}{ }^{2}+\cdots+M_{k}{ }^{2}\right)^{1 / 2} / k^{1 / 2}$.

- Arc length: the average of the absolute differences between all adjacent magnitude values in a window, i.e., $\quad\left(\left|M_{2}-M_{1}\right|+\left|M_{3}-M_{2}\right|+\cdots+\mid M_{k}-\right.$ $\left.M_{k-1} \mid\right) /(k-1)$. Intuitively, this captures the average of the first derivative of the acceleration.

- Product of arc length and standard deviation: the product of the two feature values.

Because the accelerometer sensor reports readings in the same units on all Android phones, and because our features are relatively simple, we believe these features capture fundamental, device-independent characteristics of the motion rather than anything specific to the particular device used for data capture (e.g., in contrast to the hardware-based differences documented by Dey et al. [6]).

We obtained 120 positive instances from the 120 simulated thefts collected on a Nexus $5 X$. After applying the $40 \mathrm{~m} / \mathrm{s}^{2}$ threshold, we obtained approximately 248,000 negative samples from the data collected in the field study. We then applied Boolean classification techniques to this data set. We also obtained an additional data set of 120 positive instances from the 120 simulated thefts collected on a Nexus 6P. We used this additional data set to test the generalizability of our theft detector.

\subsection{Machine Learning Algorithms}

We evaluate three standard machine learning algorithms: linear SVM, logistic regression and random forests. Because we have many more negative samples than positive ones, we tried different settings for class weights to weight positive instances more highly than negative ones. We also evaluated different window sizes for the after-window. We found that a 2-second after-window yielded better accuracy than a 1-second after-window since 2-second windows encapsulate more complete acceleration information about the motions, and larger window sizes did not offer much improvement. Therefore, all of our experiments use a 1-second before-window and a 2 -second after-window. We partitioned the entire dataset, which consists of 120 positive samples and approximately 248,000 negative samples, into training and test sets with 7:3 ratio. Then we trained the classifiers on the training set and reported the prediction results of the test set.

\section{RESULTS}

Among the three classifiers, logistic regression performs the best. Confusion matrices for logistic regression, random forests, and a linear SVM are shown in Table 2. The logistic regression classifier has a false positive rate of $0.09 \%$. Given that the field study involved $53 * 3=159$ person-weeks of data, and the logistic regression would report $248,000 * 0.09 \%=223$ false positives (the number of samples extracted from the field study data times the false positive rate). Thus, on average, users would receive $223 / 159 \approx 1.4$ false alarms every week, and a true positive rate of $100 \%$. The random forests classifier has an even lower false positive rate $(\approx 1$ false alarm per month), but only detects $60 \%$ of the thefts.

Only a small fraction of predicted positives will actually be theft, but we expect this will be acceptable due to the relatively low cost of false positives: it means users will have to unlock their phones one extra time per week, which will likely be tolerable. We report the false positive rate rather than precision, because the false positive rate is easily interpretable and not sensitive to the rate at which theft occurs. The precision is sensitive to the number of simulated thefts included in the dataset, which might not match the number of actual thefts that are likely to occur over a given period of time.

We also tested our classifiers on a set of 120 positive data points collected using a Nexus 6P. A detection rate of $98.3 \%$ indicates that our detector works on other smartphone models.

To get a better understanding of why our classifier is successful, we computed feature rankings to find the most predictive features. For logistic regression, we used standardized coefficients as the feature importance score: the score for feature $i$ is $\left|\alpha_{i}\right| \cdot \sigma_{i}$, where $\alpha_{i}$, is the coefficient for feature $i$ in the logistic regression model, and $\sigma_{i}$ is the standard deviation of feature $i$ in the training set. In addition, we computed the $95 \%$ confidence intervals for all standardized coefficients using 50 epochs of the entire data set. The feature importance scores and their $95 \%$ confidence intervals for the logistic regression classifier are listed in Table 3. The most discriminative features are the root mean square and mean of the before-window, the root mean square and mean of the afterwindow, and the standard deviation of the after-window. Plotting a histogram of feature values (see Figures 3 and 4), we can see that those features do appear to provide good discrimination.

Table 2. Confusion matrices for a logistic regression classifier (at top; with class weights 1:200), random forests classifier (middle; class weights 1:5000), and a linear SVM classifier (bottom; class weights 1:1000).

\begin{tabular}{|c|c|c|}
\hline & Predicted Negative & Predicted Positive \\
\hline True Negative & 74446 & 72 \\
\hline True Positive & 0 & 37 \\
\hline
\end{tabular}




\begin{tabular}{|c|c|c|}
\hline & Predicted Negative & Predicted Positive \\
\hline True Negative & 74503 & 15 \\
\hline True Positive & 14 & 23 \\
\hline
\end{tabular}

\begin{tabular}{|c|c|c|}
\hline & Predicted Negative & Predicted Positive \\
\hline True Negative & 74501 & 17 \\
\hline True Positive & 17 & 20 \\
\hline
\end{tabular}

Table 3. Feature importances and their $95 \%$ confidence intervals for logistic regression. (b) denotes the features extracted from the 1s window before the 40-spike; (a) denotes the features extracted from the 2 s window after the 40 -spike.

\begin{tabular}{|l|r|}
\hline Feature & Feature Importance \\
\hline Root mean square (b) & $143.8786849( \pm 0.08714717)$ \\
\hline Mean (b) & $129.8910092( \pm 0.08033373)$ \\
\hline Root mean square (a) & $93.8382532( \pm 0.04439512)$ \\
\hline Mean (a) & $88.1273891( \pm 0.03972303)$ \\
\hline Standard deviation (a) & $66.7204655( \pm 0.02228971)$ \\
\hline Standard deviation (b) & $47.7685313( \pm 0.03349549)$ \\
\hline Arc length (a) & $27.3736785( \pm 0.01463394)$ \\
\hline Maximum (b) & $2.73231469( \pm 0.00041407)$ \\
\hline Arc length * SD (a) & $2.69875036( \pm 0.00097762)$ \\
\hline Arc length (b) & $1.16578948( \pm 0.00855526)$ \\
\hline Maximum (a) & $0.05985700( \pm 0.00065551)$ \\
\hline Arc length * SD (b) & $0.04950325( \pm 0.00090281)$ \\
\hline
\end{tabular}

We also rank the features for the random forests classifier using scikit-learn's feature importance score, which estimates the relative importance of the features by computing the expected fraction of the samples they contribute to. Thus the higher in the tree, the more important the feature is [14]. We also computed the $95 \%$ confidence intervals for all feature importance scores using 50 epochs of the entire data set. The resulting feature importance scores and their $95 \%$ confidence intervals for the random forests classifier are listed in Table 4.

To compare the performance of random forests and logistic regression, we fine tuned the class weights for the logistic regression classifier to lower its true positive rate until it is approximately the same as the random forests classifier, then we compared the number of false positive instances of the two classifiers. We find that logistic regression has 14 false positive instances at a $67 \%$ true positive rate (25 true positive instances), while random forests has 15 false positive instances at a $62 \%$ true positive rate (23 true positive instances). Thus the performance of the two classifiers seems comparable in this regime. The advantage of logistic regression is that we found adjusting class weights was more effective at controlling the false-positive/falsenegative tradeoff for the logistic regression classifier. The Receiver Operating Characteristic (ROC) curves for the logistic regression and random forests classifiers are shown in Figure 5.

\section{CONCLUSION}

In this work, we demonstrate that accelerometer data is enough to detect some common forms of smartphone theft, such as pickpocket and grab-and-run, without sacrificing usability by inundating the user with false alarms. It is remarkable that machine learning is so effective and can detect $100 \%$ of our simulated thefts. We suspect that this is because the kinds of thefts we consider here involve a rapid jerking motion followed by the thief running away, which induces a unique pattern in the accelerometer sensor readings.

Table 4. Feature importances and their $95 \%$ confidence intervals for random forests. (b) denotes the features extracted from the 1s window before the 40-spike; (a) denotes the features extracted from the $2 \mathrm{~s}$ window after the 40 -spike.

\begin{tabular}{|l|l|}
\hline Feature & Feature Importance \\
\hline Root mean square (a) & $0.24251892( \pm 0.00734018)$ \\
\hline Mean (a) & $0.19351276( \pm 0.00685086)$ \\
\hline Standard deviation (a) & $0.17634030( \pm 0.00601571)$ \\
\hline Maximum (a) & $0.10975278( \pm 0.00480411)$ \\
\hline Arc length * SD (a) & $0.07541471( \pm 0.00382659)$ \\
\hline Arc length (a) & $0.04821400( \pm 0.00264303)$ \\
\hline Maximum (b) & $0.04740376( \pm 0.00172894)$ \\
\hline Arc length * SD (b) & $0.03291854( \pm 0.00128944)$ \\
\hline Standard deviation (b) & $0.02828350( \pm 0.00115971)$ \\
\hline Arc length (b) & $0.02072662( \pm 0.00103551)$ \\
\hline Root mean square (b) & $0.01544474( \pm 0.00078121)$ \\
\hline Mean (b) & $0.00946937( \pm 0.00077147)$ \\
\hline
\end{tabular}

We envision that a smartphone could run our classifier continuously and automatically lock the phone whenever a theft is suspected. We expect that the inconvenience of unlocking the phone once more per week would be tolerable, and might not even be noticed by users. If combined with other heuristics to reduce the false positive rate further (e.g., the phone is not unlocked within a short period after the suspected theft; the phone moves to some new location it has never been before), it might be possible to notify the owner or take other measures as well, when a theft is detected.

As mentioned earlier, $40 \%$ of smartphone users do not lock their phone with a PIN or passcode [7,9], so currently thieves have full access to the personal data of these users. We envision our solution would help protect those users: if a suspected theft is detected, the phone could lock itself so it requires a PIN or passcode to unlock; this way, users would only need to enter a PIN or passcode about once a week, rather than every time they want to use the phone. For the $60 \%$ of smartphone users who do have a PIN or passcode enabled, our scheme might have less benefit. Because the phone can immediately detect the theft and lock itself, it prevents thieves who grab the phone while it is unlocked from accessing the user's data, but it is unnecessary against thieves who steal the phone while it is locked (e.g., pickpocket theft). Our scheme might also be helpful for finding 
the stolen phone: if a suspected theft is detected, the phone could enable GPS, start tracking its location, upload its location to a cloud server in real-time, and continue until the phone is unlocked (if the user has not already enabled a "find my phone" feature).

We expect that our solution would have negligible impact on battery life and phone performance. Modern phones support batched accelerometer sensing, where the accelerometer hardware buffers sensor readings, so the application CPU only has to wake up to read sensor data when the buffer is full. As a result, it is possible to record accelerometer sensor values at high sampling rates with negligible power draw. Moreover, thanks to the prefiltering (the $40 \mathrm{~m} / \mathrm{s}^{2}$ threshold), we only need to apply the classifier on a tiny fraction of time windows (only about 10 times

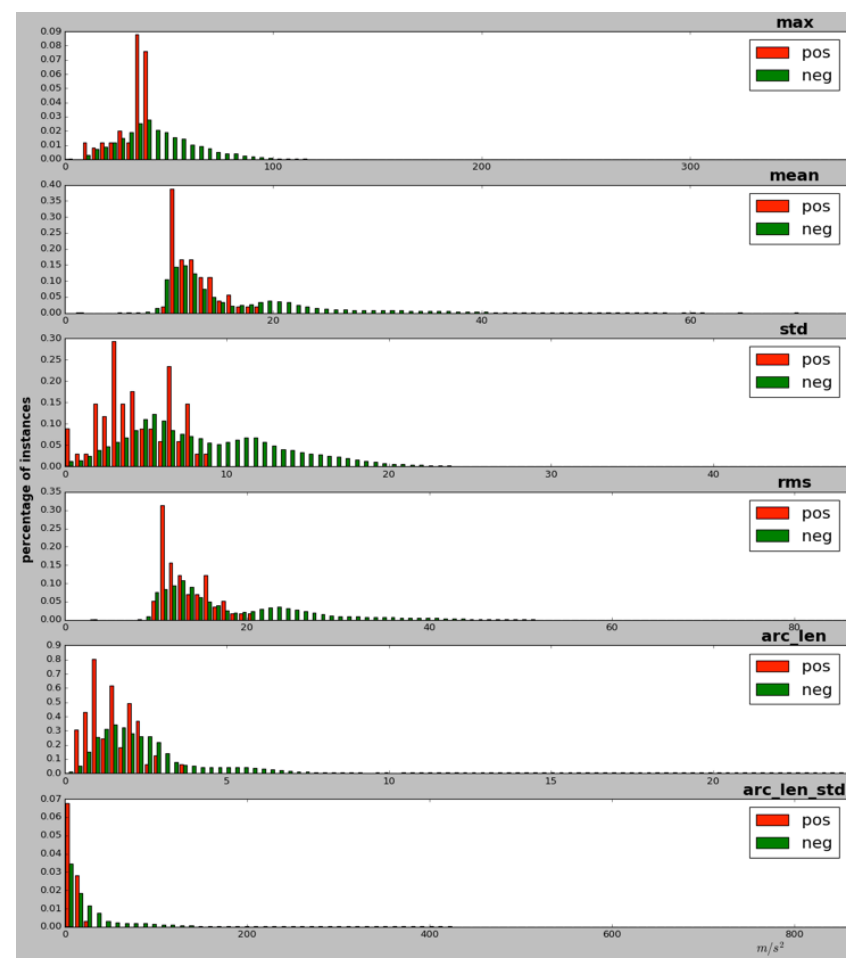

Figure 3. Histogram for each of the 6 features for the 1-second window before the $40 \mathrm{~m} / \mathrm{s}^{2}$ spike. The features are listed in the order presented in Section 3.2, e.g., the top histogram is for the maximum. Red bars indicate thefts, and green bars indicate non-theft windows.

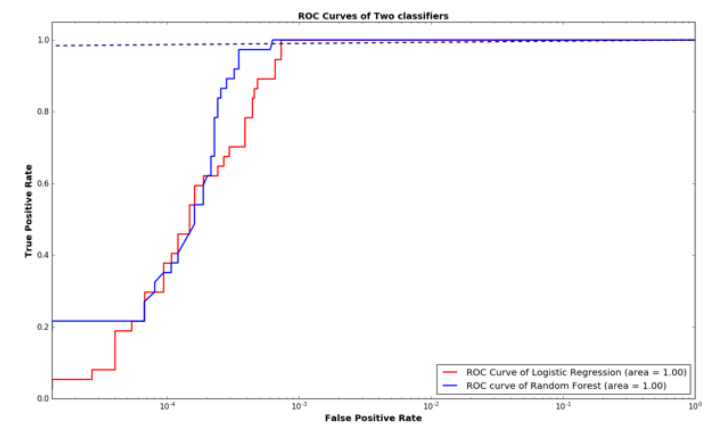

Figure 5. ROC curves of logistic regression and random forests. The $x$-axis is in logarithmic scale. per hour on average), so the impact on battery life should be negligible.

The primary limitation of our work is that we work with simulated thefts. It is difficult to obtain accelerometer data on actual theft occurring in the wild, but perhaps a practical deployment could obtain such data, and then use it to further train the classifiers.

It may be possible to improve our results further by using other sensors on the smartphone, such as the step counter. The biggest open question is whether our methods can be extended to a more diverse set of theft scenarios; we hope that our work will inspire others to investigate this direction further.

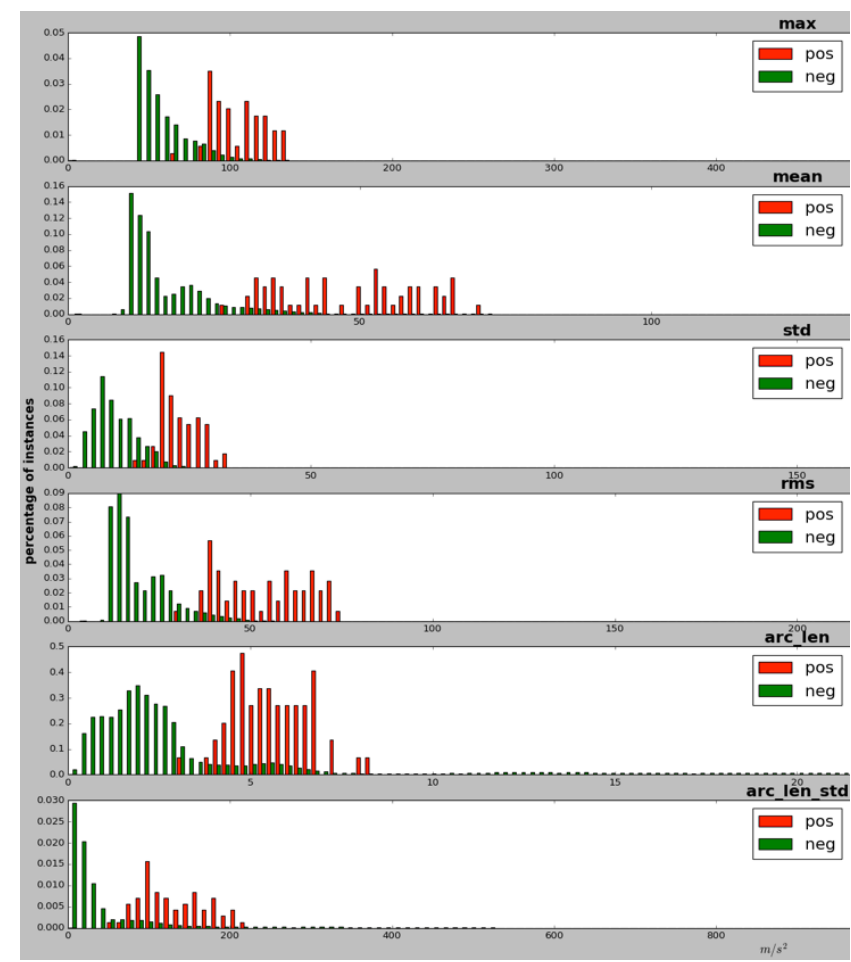

Figure 4. Histogram of feature values in the 2-second window after the $40 \mathrm{~m} / \mathrm{s}^{2}$ spike.

\section{ACKNOWLEDGMENTS}

The authors would like to thank Prakash P. Bhasker and Micah J. Sheller for providing us with the Android sensor monitoring software, Jennifer Chen from Good Research for her assistance on conducting the user study, and Irwin Reyes, David Fifield and Michael McCoyd for giving feedback on our paper drafts. This research was conducted at The Intel Science and Technology Center for Secure Computing (http://scrub.cs.berkeley.edu/) at UC Berkeley and was also supported by the National Science Foundation under award CNS-1514457.

\section{REFERENCES}

[1] 2017. Android Developers Sensor Manager https://developer.android.com/reference/android/hardware/Se nsorManager.html.

[2] Jan Lauren Boyles and Aaron Smith and Mary Madden. 2012. Privacy and Data Management on Mobile Devices. http://www.pewinternet.org/2012/09/05/privacy-and-datamanagement-on-mobile-devices/ (September 5, 2012). 
[3] Shan Chang and Ting Lu and Hui Song. 2016. SmartDog: Real-time Detection of Smartphone Theft. In Internet of Things (iThings) and IEEE Green Computing and Communications (GreenCom) and IEEE Cyber, Physical and Social Computing (CPSCom) and IEEE Smart Data (SmartData), 2016 IEEE International Conference on. IEEE.

[4] Calla Deitrick. 2015. Smartphone thefts drop as kill switch usage grows.

http://www.consumerreports.org/cro/news/2015/06/smartpho ne-thefts-on-the-decline/index.htm (June 11, 2015).

[5] Mohammad O. Derawi and Claudia Nickel and Patrick Bours and Christoph Busch. 2010. Unobtrusive UserAuthentication on Mobile Phones using Biometric Gait Recognition. In Intelligent Information Hiding and Multimedia Signal (IIH-MSP) Sixth International Conference Processing. IEEE, 306-311

[6] Sanorita Dey and Nirupam Roy and Wenyuan Xu and Romit Roy Choudhury and Srihari Nelakuditi. 2014. AccelPrint: Imperfections of Accelerometers Make Smartphones Trackable. In Proceedings of the 20th Annual Network and Distributed System Security Symposium (NDSS '14).

[7] Serge Egelman and Sakshi Jain and Rebecca S. Portnoff and Kerwell Liao and Sunny Consolvo and David Wagner. 2014. Are You Ready to Lock? Understanding User Motivations for Smartphone Locking Behaviors. In Proceedings of 2014 ACM SIGSAC Conference on Computer and Communications Security. ACM SIGSAC, 750-761.

[8] Tao Feng and Xi Zhao and Weidong Shi. 2013. Investigating Mobile Device Picking-up Motion as a Novel Biometric Modality. Biometrics: Theory, Applications and Systems (BTAS), 2013 IEEE Sixth International Conference on. IEEE
[9] Harbach, Marian and De Luca, Alexander and Malkin, Nathan and Egelman, Serge. 2016. Keep on Lockin' in the Free World: A Multi-National Comparison of Smartphone Locking. In Proceedings of 2016 CHI Conference on Human Factors in Computing Systems (CHI '16). ACM, New York, NY, USA 4823-4827. http://doi.acm.org/10.1145/2858036.2858273.

[10] Felix Juefei-Xu and Chandrasekhar Bhagavatula and Aaron Jaech and Unni Prasad and Marios Savvides. 2012. Gait-ID on the Move: Pace Independent Human Identification Using Cell Phone Accelerometer Dynamics. In Biometrics: Theory, Applications and Systems (BTAS), 2012 IEEE Fifth International Conference on. IEEE.

[11] Jennifer R. Kwapisz and Gary M. Weiss and Samuel A. Moore. 2010. Cell Phone-Based Biometric Identification. In Biometrics: Theory Applications and Systems (BTAS), 2010 Fourth IEEE International Conference on. IEEE.

[12] Shrirang Mare and Andres Molina-Markham and Cory Cornelius and Ronald Peterson and David Kotz. 2014. ZEBRA: Zero-Effort Bilateral Recurring Authentication. In Security and Privacy (SP), 2014 IEEE Symposium on. IEEE, 705-720.

[13] Abena Primo and Vir V. Phoha and Rajesh Kumar and Abdul Serwadda. 2014. Context-Aware Active Authentication Using Smartphone Accelerometer Measurements. In Proceedings of the IEEE Conference on Computer Vision and Pattern Recognition Workshops. IEEE, 98-105.

[14] scikit-learn. 2016. 3.2.4.3.1. sklearn.ensemble.RandomForestClassifier. http://scikitlearn.org/stable/modules/generated/sklearn.ensemble.Rando mForestClassifier.html (2016). 